\title{
Optical metrology for immersed diffractive multifocal ophthalmic intracorneal lenses
}

\author{
P. Tankam \\ T. Lépine \\ thierry.lepine@institutoptique.fr

\section{F. Castignoles} \\ P. Chavel
}

\author{
Laboratoire Hubert Curien (UMR 5516 CNRS), Université de Lyon, Université Jean-Monnet, 42000, \\ Saint-Etienne, France \\ Laboratoire Hubert Curien (UMR 5516 CNRS), Université de Lyon, Université Jean-Monnet, 42000, \\ Saint-Etienne, France \\ Institut d'Optique Rhône-Alpes 18 rue Benoît Lauras 42000, Saint-Etienne, France \\ Presbeasy, Lyon, 69007, France
}

Laboratoire Charles Fabry, Université Paris-Sud, CNRS, Palaiseau, France

This paper deals with the optical characterization of diffractive multifocal Intra-Corneal Lenses (ICLs) that we have developed in order to correct presbyopia. These diffractive multifocal lenses are made of a very soft material (permeable to oxygen and nutrients), with a thickness smaller than $100 \mu \mathrm{m}$ and require liquid immersion. As a consequence, most of the conventional metrology methods are unsuited for their characterization. We developed specific setups to measure diffractive efficiencies and Modulation Transfer Function (MTF) adapted to such components. Experimental results are in good agreement with Zemax ${ }^{\circledR}$ simulations. For the best of our knowledge, it is the first time that optical characterization is devoted to the ICLs. Furthermore, most of the IOL's optical characterizations are focused on far vision MTF and don't assess the near vision MTF, which we study in this paper.

[DOI: http://dx.doi.org/10.2971/jeos.2012.12037]

Keywords: Diffractive lenses, optical design and fabrication, modulation transfer function, metrology, ophthalmology

\section{INTRODUCTION}

Bifocal diffractive intraocular lenses (IOLs) are nowadays widely used in the correction of presbyopia or when cataract surgery is performed [1, 2]. Such lenses can be obtained by combining two lenses, a carrier lens which determines the power for the far vision and a diffractive profile providing the addition needed to correct the near vision [3]. However, intraocular correction of presbyopia leads to heavy surgical operations which are invasive and irreversible. To solve these issues, we proposed a new concept of presbyopia correction based on bifocal diffractive Intra-Corneal Lenses (ICLs). The surgical operation will become easier, less-invasive and reversible, since it will be conducted in the cornea and it won't need removing the crystalline lens. Such an ICL consists of a plane carrier lens with an infinite focal length in order to keep unchanged the far vision power and a diffractive bifocal profile with an addition of 2.5D which is used to correct the near vision. In our previous studies [4], we designed and compared four different profiles of diffractive lenses, in term of efficiency, Modulation Transfer Function (MTF) and chromatism. The simulations of these properties with Matlab and Zemax ${ }^{\circledR}$ have shown that the parabolic profile presents the better performance for our purpose [4]. We designed this profile to yield $70 \%$ of absolute efficiency in far vision (the 0th order) and $16 \%$ in near vision (the $-1^{\text {st }}$ order) at the design wavelength of $550 \mathrm{~nm}$. Figure 1 shows the profile (Figure 1(a)) as well as an image of our diffractive lens (Figure 1(b)).
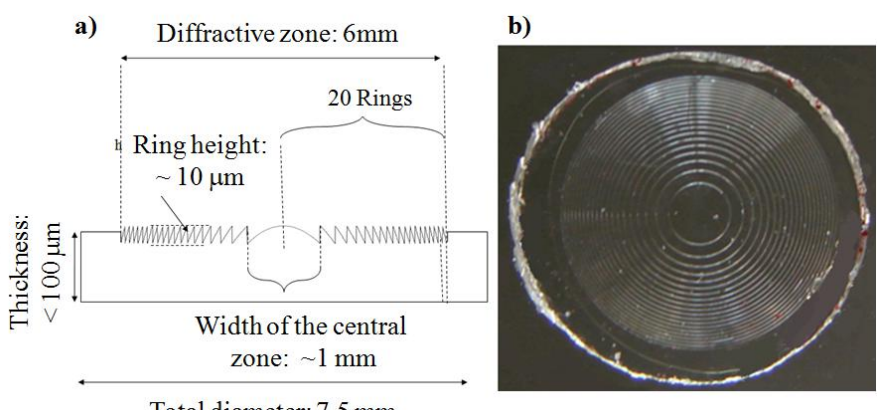

Total diameter: $7.5 \mathrm{~mm}$

FIG. 1 Diffractive lens. a) Profil. b) 2D imaging.

Figure 2 illustrates the principle of correction of prebyopia with a bifocal diffractive ICL.

We assume that, without the lens, the patient has a perfect far vision and a poor near vision. The addition of the lens leads to a better near vision, while in return the far vision is slightly degraded due to the halo caused by the $-1^{\text {st }}$ order. This compromise could not be avoided for multifocal correction $[5,6]$.

In order to control the performance of our diffractive lens, we carried out its experimental characterization in terms of diffractive efficiencies and MTF measurements. One major constraint in this characterization is related to the fact that the 


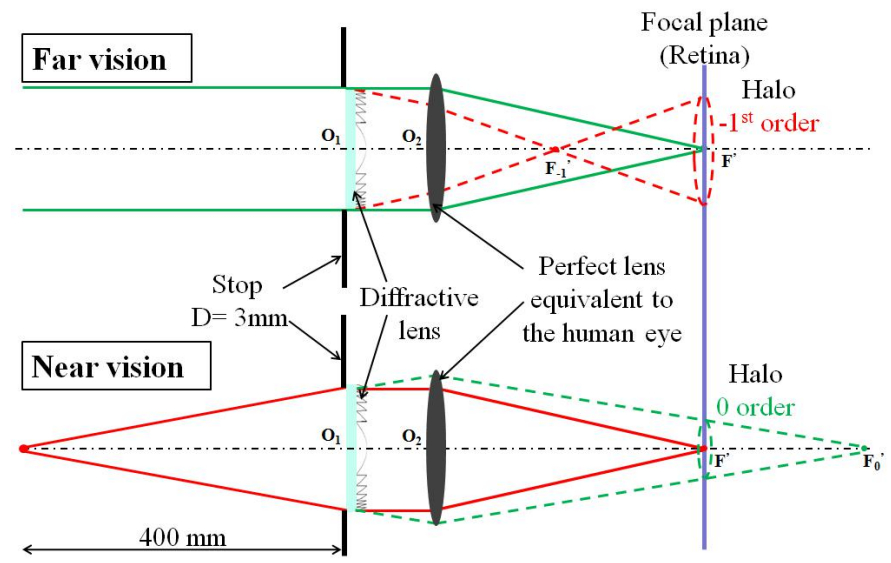

FIG. 2 Principle of correction of presbyopia with a bifocal lens.

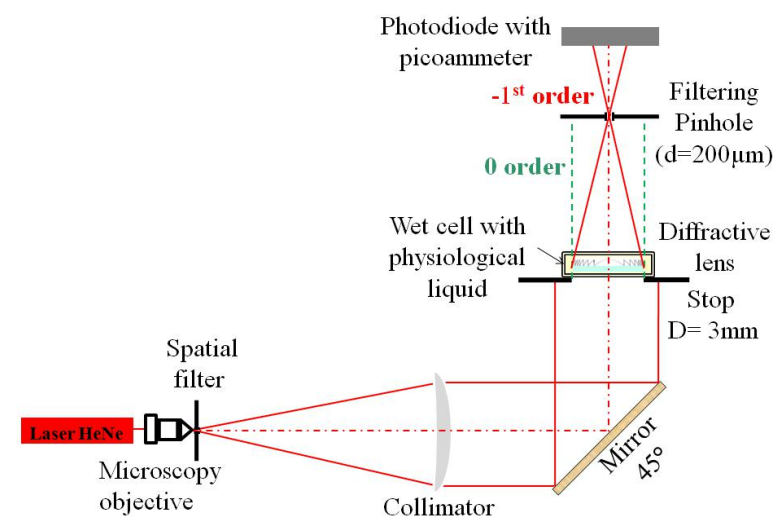

FIG. 3 Diffractive efficiency measurement (for the $-1^{\text {st }}$ order).

lens must be immersed in a liquid with a refractive index close to that of cornea, because deshydratation distorts our implant when it is in the air.

\section{DIFFRACTIVE EFFICIENCIES MEASUREMENT}

The diffractive efficiency of each order is equal to the ratio between the diffracted energy in this order and the total energy incident on the profile. Figures 3 and 4 show the schematic diagrams of the setups developed for efficiency measurements of the $-1^{\text {st }}$ and $0^{\text {th }}$ orders.

The HeNe laser at $633 \mathrm{~nm}$ is spatially filtered and collimated to yield a smooth and flat wavefront. The beam diameter is stopped to $3 \mathrm{~mm}$, close to that of the pupil of a 50 year old patient in daylight. Since the lens floats in its cell, the optical bench must be vertical. The $0^{\text {th }}$ order remains collimated behind the diffractive lens and is brought to focus by using an additional convergent lens of $100 \mathrm{~mm}$ focal length (Figure 4). As detailed below, this lens, which has a high Strehl ratio $(98 \%)$, is placed at the focus of the $-1^{\text {st }}$ order to reduce its contribution in the $0^{\text {th }}$ order efficiency measurement. The $-1^{\text {st }}$ order focuses at $400 \mathrm{~mm}$ at the design wavelength $(550 \mathrm{~nm})$, which corresponds to $347 \mathrm{~mm}$ at the illuminating wavelength of these setups $(633 \mathrm{~nm})$. For each focus, the energy is integrated through a pinhole whose diameter is equal to that of the first ring of the Airy pattern defined by $d=2.44 \lambda f_{o b} / D$;

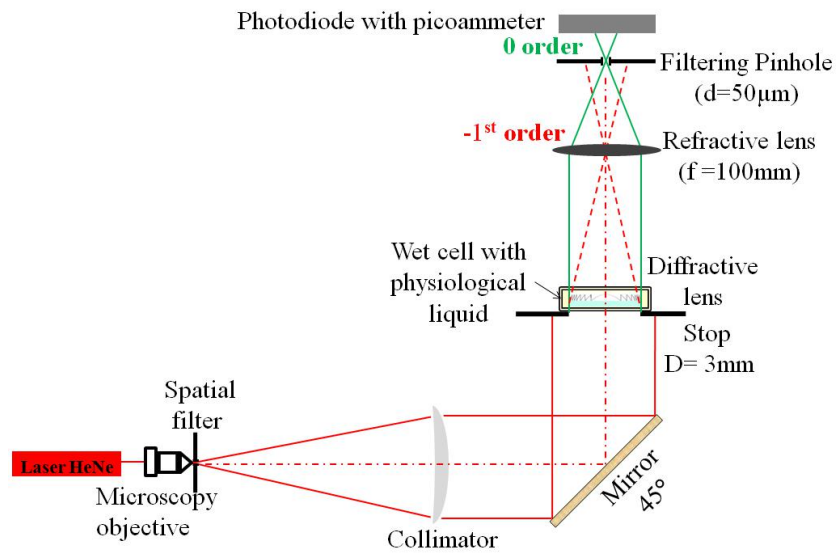

FIG. 4 Diffractive efficiency measurement (for the $0^{\text {th }}$ order).

\begin{tabular}{lll}
$\lambda=633 \mathrm{~nm}$ & $\begin{array}{l}\text { Experimental } \\
\text { Efficiencies }\end{array}$ & $\begin{array}{l}\text { Simulated } \\
\text { Efficiencies }\end{array}$ \\
\hline The $0^{\text {th }}$ order & $70 \pm 3 \%$ & $78 \%$ \\
The $1^{\text {st }}$ order & $14 \pm 3 \%$ & $12 \%$ \\
\hline
\end{tabular}

TABLE 1 Comparison of experimental and simulated diffractive efficiencies.

with the wavelength of the illuminating beam $\lambda=633 \mathrm{~nm}$, the diameter of the stop $D=3 \mathrm{~mm}$ and the focal length associated with the diffracted order $f_{o b}\left(100 \mathrm{~mm}\right.$ for the $0^{\text {th }}$ order and $347 \mathrm{~mm}$ for the $-1^{\text {st }}$ order). It is important to notice that the first ring of an Airy pattern contains $84 \%$ of the total energy if there is no aberration; this assumption leads to a correction factor which must be taken into account to calculate the diffractive efficiency. The energy is measured with a large silicon photodiode $(1 \mathrm{~cm}$ in diameter) associated with a picoammeter (Keithley 485).

Experimental efficiencies agree reasonably well with the simulated efficiencies which are given here at $633 \mathrm{~nm}$ (Table 1), although the 0th order efficiency simulated is slightly out of tolerance range of the experimental efficiency. This can result from a difference between the profile height used in the simulation and the experimental one. However, no mechanical profilometer can be used in this case because the material is too soft. Furthermore, the slight refractive index difference between the implant and its liquid environment makes it difficult to reach the surface profile with an optical profilometer due to a poor fringes contrast. This aspect is currently under investigation.

\section{MTF MEASUREMENT}

The MTF is a well-known concept to evaluate the performance of optical systems [7]. We have assessed different methods to calculate the MTF such as wavefront measurement methods $[8,9]$. Particularly, we have shown that Shack-Hartmann sensors are hampered by the apparition of multiple spots [10]. Another method is based on the measurement of the Point Spread Function (PSF). Zhang et al. have demonstrated the suitability of this method for the characterization of thermopneumatically tunable micro-lenses [11]. Koronkevich et al. have also used this method for bifocal diffractive-refractive 


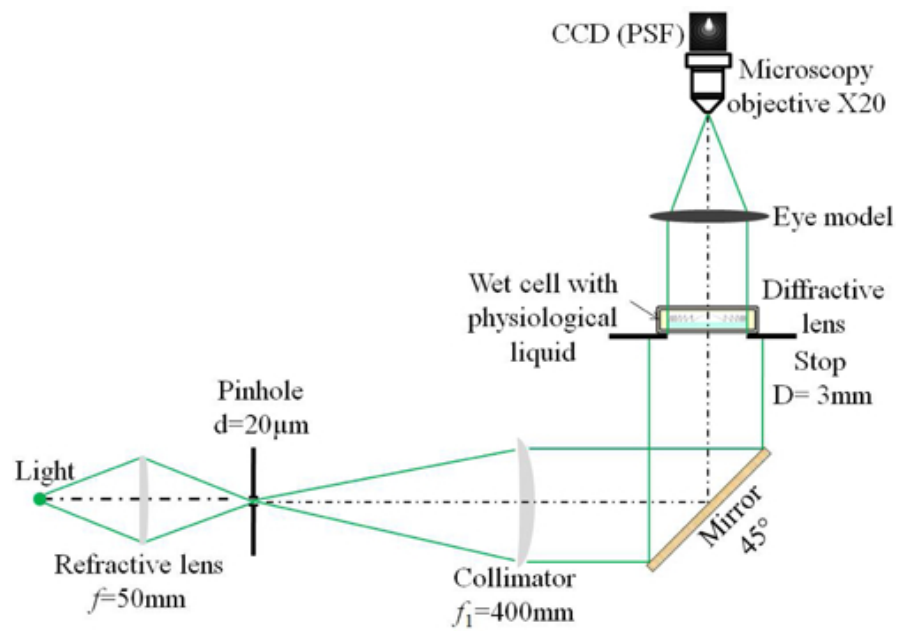

FIG. 5 PSF setup in far vision.

IOLs [12]. Due to the constraints related to the material and the geometry of our lens, we have chosen to develop a PSF setup, emphasizing the selection of critical parameters such as the size of the point source and the sampling of the PSF. Figure 5 presents the experimental setup in its far vision setting.

The setup for the near vision is obtained by removing the collimator and putting the object (pinhole) at the distance of about $400 \mathrm{~mm}$ from the diffractive lens (Figure 6). The eye is modeled by an achromat of high quality (Strehl ratio of $92 \%$ at $633 \mathrm{~nm}$ ) with a power (51D) close to that of the human eye (59D). All PSF measurements were done using a green filter (Flat transmission close to $100 \%$ between $500 \mathrm{~nm}$ and $580 \mathrm{~nm}$ ). Theoretical studies of the chromatism of our implant can be found in [4]. From the above considerations, the design of the bench should meet the following rules.

- First of all, the image of the pinhole given by the optical system (diffractive lens + eye model) must be smaller than the Airy pattern of this optical system. Thus, the diameter $\mathrm{d}$ of the pinhole must fulfill the condition $d \leq \lambda f_{1} / D$. We chose in our case $d=20 \mu \mathrm{m}$ ( $\lambda=0.55 \mu \mathrm{m}, D=3 \mathrm{~mm}$ and the focal length of the collimator $f_{1}=400 \mathrm{~mm}$ ).

- Next, the sampling of the image by the CCD camera must respect the Shannon condition. Given the pixel size $\left(p_{x}=9 \mu \mathrm{m}\right)$, the image of the pinhole given by the optical system (diffractive lens + eye model) must be magnified by a factor $g_{y}$ such that $g_{y} \geq 2 D p_{x} / \lambda f_{2}$. where $f_{2}=19.6 \mathrm{~mm}$ is the focal length of the eye model. In our bench, we use $g_{y}=20$.

- Finally, the CCD must have a high dynamic range in order to correctly sample the Airy pattern and the much weaker halos induced by the parasitic orders. Two different CCD cameras were tested: a Sony video camera (XCD-SX 910, 8 bits output) and a cooled CCD designed for astronomy (SBIG ST8 XME, 16 bits output). Due to its higher dynamic range, the SBIG camera allowed us to record the whole PSF (central peak and halos) in a single image.

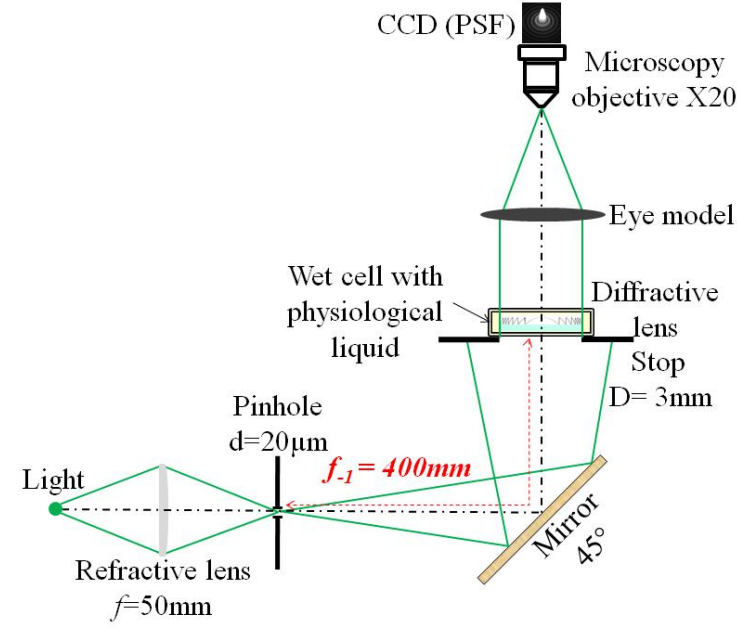

FIG. 6 PSF setup in near vision.

The MTF are obtained by calculating the normalized modulus of the Fourier transform of the PSF. Care must be taken with the CCD dark level which contributes to a wrong normalization of the MTF. This problem was solved by carrying out the experiments in darkness and subtracting from the PSF image the mean of a small zone chosen far from the PSF peak. Indeed, we have compared different ways to remove the background in the image including the recording of the true dark level [2] but the method proposed here is more convenient since the camera sensor is large enough to consider a zone far from the PSF area to determine the dark level. We have checked that the choice of this zone does not alter the results of the MTF provided that it is sufficiently far from the PSF peak. Our Matlab code automatically detects the PSF peak and automatically chooses the furthest zone from the PSF as the dark level. Thus, no additional background recording is required. Finally, to check this setup, the MTF of the eye model alone (without the diffractive lens) obtained on our optical bench with a red filter was compared with the MTF obtained with the Zygo interferometer (GPI-XP) at $633 \mathrm{~nm}$. Very good agreement was obtained (Figure 7).

\section{RESULTS AND DISCUSSION}

We present our experimental results of MTF compared with the simulations of our diffractive lenses obtained with Zemax ${ }^{\circledR}$ [4]. Figure 8 shows the MTF measured in far and near visions.

Curves 1(a) and 2(a) present the MTF in far vision for the eye model respectively without and with the diffractive lens, whereas curves $1(b)$ and 2(b) show the MTF in near vision for the eye model respectively without and with the diffractive lens. As said above, the curves 1(a) and 1(b) correspond to a presbyopic eye which has a correct far vision and a blurred near vision. Even though the addition of the diffractive lens slightly reduces the far vision quality (curve 2(a)), it really improves the near vision (curve 2(b)). These measured MTF are compared to those simulated with the green filter of our CCD (curves 3(a) and 3(b) for far and near visions respectively). The experimental results are in good agreement with the simulations and the repeatability is better than $2 \%$. Of course, the 


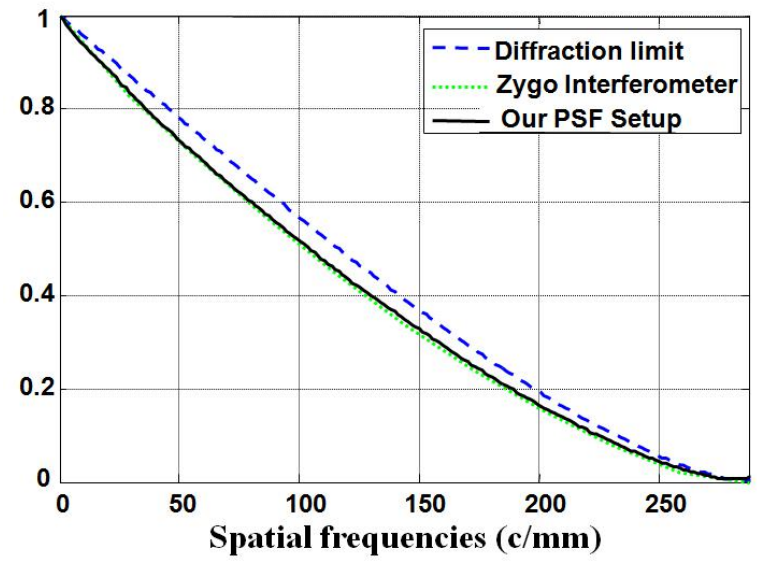

FIG. 7 MTF measurement of the eye model alone $(f=19.6 \mathrm{~mm}, D=3 \mathrm{~mm})$.

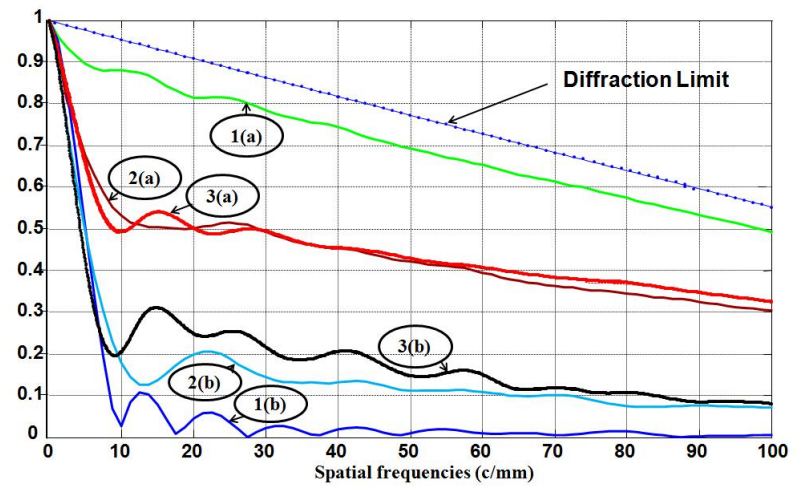

FIG. 8 Comparison between simulated and experimental MTF for the eye model in far vision (a) and near vision (b): 1 MTF measured without diffractive lens. 2 MTF measured with diffractive lens. 3 MTF simulated with diffractive lens.

difference between the simulations and experimental results is about $8 \%$ for near vision, but this difference is compatible with the acceptable precision for MTF measurement (repeatability: $9 \%$ and reproducibility: $19 \%$, according to the ophthalmic ISO 11979-2 standard for IOL metrology). As mentioned at the end of the Section 2, this difference could be reduced by taking into account the real value of the profile height in the simulation. Finally, we can see that our ICL enables to gain about $20 \%$ in MTF in near vision while maintaining sufficient MTF in far vision [13]. Furthermore, it restores the modulus and phase within the vision bandwidth of the patient, which is very important for the resolution limit of our system.

Note that with regards to the specific properties of our implant (intracorneal, soft material), commercial instruments are not adapted for their characterization.

Finally, the MTF of our implant in far vision is close to that of the IOLs $[14,15]$. The comparison is hard to do for near vision as, to our knowledge, there is no published evaluation for IOLs.

\section{CONCLUSION}

In summary, this paper presents, to the best of our knowledge, the first optical characterization of a novel diffractive bifocal intracorneal lens designed to correct presbyopia. The concept of the intracorneal implant is innovative and will make surgical correction of the presbyopia an easier and reversible operation. The optical characterization proposed is suitable for this type of component (multifocal, soft material, small size and immersed in a liquid) and provides good results for diffractive efficiency and MTF measurements consistent with our modeling. It turn out that the tested lens appears to be a promising solution for the correction of presbyopia and we are working currently in reducing our inlays thickness.

\section{ACKNOWLEDGEMENTS}

Patrice Tankam would like to thank Saint-Etienne Métropole for its financial support.

\section{References}

[1] J. A. Davison, and M. J. Simpson, "History and development of the apodized diffractive intraocular lens," J. Cataract Refr. Surg. 32(5), 849-858 (2006).

[2] M. J. Simpson, "Diffractive multifocal intraocular lens image quality," Appl. Optics 32(19), 3621-3626 (1992).

[3] A. L. Cohen, "Diffractive bifocal lens designs," Optom. Vis. Sci. 6, 461-468 (1993).

[4] F. Castignoles, M. Flury, T. Lépine, "Comparison of the efficiency, MTF and chromatic properties of four diffractive bifocal intraocular lens designs," Opt. Express, 18(5), 5245-5256 (2010).

[5] C. Vergès, "Understanding multifocal halos," in Mastering refractive IOLs: the art and science, D. F. Chang, ed., 267-272 (SLACK incorporated, New Jersey, 2008).

[6] K. L. Waltz, "Understanding multifocal halos," in Mastering refractive IOLs: the art and science, David F. Chang, ed., 273-277 (SLACK incorporated, New Jersey, 2008).

[7] M. Bass, Handbook of Optics Volume II - Devices, Measurements and Properties (Second Edition, McGraw-Hill, New York, 1995).

[8] J. Primot, "Theoretical description of Shack-Hartmann wave-front sensor," Opt. Commun. 222, 81-92 (2003).

[9] S. Velghe, J. Primot, N. Guérineau, M. Cohen, and B. Wattellier, "Wave-front reconstruction from multidirectional phase derivatives generated by multilateral shearing interferometers," 0 pt. Lett. 30, 245-247 (2005).

[10] F. Castignoles, T. Lépine, P. Chavel, and C. Cohen, "ShackHartmann multiple spots with diffractive lenses," Opt. Lett. 36(8), 1422-1424 (2011).

[11] W. Zhang, K. Aljasem, H. Zappe and A. Seifert, "Completely integrated, thermo-pneumatically tunable microlens," Opt. Express 19(3), 2347-2362 (2011).

[12] V. P. Koronkevich, G. A. Lenkova, V. P. Korol'kov, and I. A. Iskakov, "Bifocal diffraction-refraction intraocular lenses," J. Opt. Technol. 74(12), (2007).

[13] ISO 11979-2:1999 Ophthalmic implants - Intraocular lenses - Part 2: Optical properties and test methods (DIN Deutsches Institut für Normung e. V., Berlin, 2000).

[14] W. A. Maxwell, S. S. Lane, and F. Zhou, "Performance of presbyopia-correcting intraocular lenses in distance optical bench tests," J. Cataract Refr. Surg. 35(1), 166-171 (2009).

[15] http://www.ppt2txt.com/r/5f5cb268/ 